\title{
Side-by-Side Epigenetics and Genetics Share Importance in Cancer Development
}

Ana Paula de Souza-Pardo*

Department of Morphology, School of Dentistry of Piracicaba, University of Campinas, Brazil

*Corresponding author: Souza-Pardo APD, Department of Morphology, School of Dentistry of Piracicaba, University of Campinas Limeira Avenue, 901, 13414-018 Piracicaba-SP Brazil, Tel: 55-19-21065381; E-mail: anapaulapardo@fop.unicamp.br

Rec Date: October 09, 2014; Acc Date: October 14, 2014 Pub Date: October 16, 2014

Copyright: ( 2015 Souza-Pardo APD. This is an open-access article distributed under the terms of the Creative Commons Attribution License, which permits unrestricted use, distribution, and reproduction in any medium, provided the original author and source are credited.

\section{Editorial}

Recently, I had in my hands an interesting and highly recommended review from Jueng Soo You and Peter Jones [1] entitled "Cancer Genetics and Epigenetics: Two Sides of the Same Coin?" which was published in Cancer Cell. In this review, the authors discuss the role and the weight of genetics and epigenetics in tumorigenesis. According to them "intriguing evidences have emerged showing that genetic and epigenetic mechanism are not separate events in cancer" and "alterations in epigenetic mechanisms can lead to genetic mutations, and genetic mutations in epigenetic regulators lead to an altered epigenome". Here, I develop this theme based on this last statement.

Cancer cannot be anymore understood as only the outcome of genetic mutations accumulation. The traditional cancer definition "set of diseases that are driven by accumulation of genetic mutations" has frustrated generations of scientists that hoped to find the cancer missing-link after the genomic decade as well as patients that patiently have waited for a miracle successful therapy. It is not that gene mutations are not relevant. Of course not! The genomic as well as the transcriptomic and the proteomic field have promoted an enormous advance in the knowledge of tumorigenesis, especially after the advent of high-throughput techniques. But maybe, we have left an important "omic" member out of the high-throughput party until recently: the epigenetics.

Apparently, for a long-time (more than 20 years have passed since the first evidences suggested an important role of epigenetics in cancer) the eyes of scientific community had been blind to this truth: epigenetic walks beside of genetics in cancer and they are not disconnected events working in a two-way road. Completely different of what had been thought, epigenetics is not a separate mechanism or a consequent event in cancer. The outcomes from the whole exomesequencing of thousands of human cancers have shown that both genetics and epigenetics share responsibilities in cancer initiation, progression and expansion. Lots of mutations have the potential to disrupt ordinary epigenetic patterns once have disturbed epigenetic regulatory genes. In turn, epigenetics is implicated in driving mutations. The presence of methylation of cytosine in the germline at CpG sites is the reason for the decrease of approximately $75 \%$ in the frequency of $\mathrm{CpG}$ sites in whole genome [1]. The spontaneous deamination of 5 -methylcytosine $(5 \mathrm{mC})$ to thymine is responsible for high levels of $\mathrm{C}$ to $\mathrm{T}$ transition mutations and as many as $50 \%$ of p53 single nucleotide changes have occurred at such sites in colon cancer, being $5 \mathrm{mC}$ directly implicated with these hotspots in somatic cells [1].

The epigenetics field comprises at least four mechanistic layers: post-translational histones modifications, DNA methylation, noncoding RNA and chromatin remodeling factors. All together are responsible for controlling how cells genome works. The advent of whole-genome approaches to investigate epigenetic pathways, such as the powerful epigenomic platform 450K DNA Methylation Microarray (Infinium, Illumina) and the Reduced Representation Bissulfite Sequencing (RRBS) technique, also contributed to transform our view on the epigenetics importance and allowed to come up the universe of epigenetic dataset generated from ENCODE, HUMAN EPIGENOME CONSORTIUM and BLUEPRINT projects. The epigenetic datasets associated to genomic data from several laboratories around the world can lead us to really understand cancer. One of first results from these global efforts is The Cancer Genome Atlas.

Perhaps today we are close to finding an effective treatment against cancer as we have never been before and it is possible to see on the horizon line the signals of a happy end in the fight against cancer. However, there is a long way ahead and we still have hard battles to win due to the fact the great majority of cancer diagnosis still has been done by pathologists using only light microscopy.

\section{References}

1. You JS, Jone PA (2012) Cancer Genetics and Epigenetics: Two Sides of the Same Coin? Cancer Cell. 22: 9-20. 